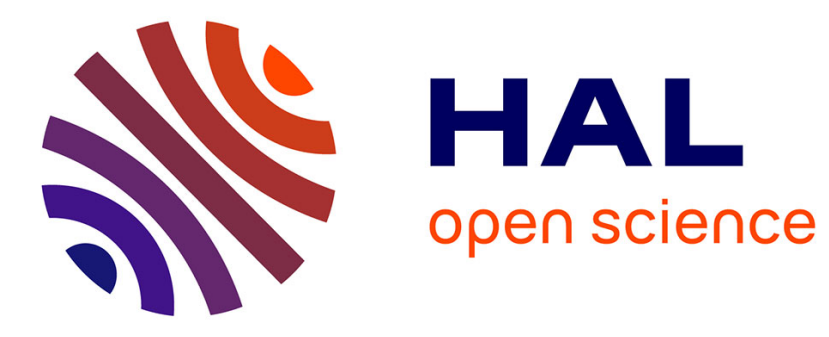

\title{
Subproblem Approach for Thin Shell Dual Finite Element Formulations
}

Vuong Quoc Dang, Patrick Dular, Ruth V. Sabariego, Laurent Krähenbühl, Christophe Geuzaine

\section{- To cite this version:}

Vuong Quoc Dang, Patrick Dular, Ruth V. Sabariego, Laurent Krähenbühl, Christophe Geuzaine. Subproblem Approach for Thin Shell Dual Finite Element Formulations. IEEE Transactions on Magnetics, 2012, 48 (2), pp.407 - 410. 10.1109/TMAG.2011.2176925 . hal-00639613

\section{HAL Id: hal-00639613 https://hal.science/hal-00639613}

Submitted on 16 Feb 2012

HAL is a multi-disciplinary open access archive for the deposit and dissemination of scientific research documents, whether they are published or not. The documents may come from teaching and research institutions in France or abroad, or from public or private research centers.
L'archive ouverte pluridisciplinaire HAL, est destinée au dépôt et à la diffusion de documents scientifiques de niveau recherche, publiés ou non, émanant des établissements d'enseignement et de recherche français ou étrangers, des laboratoires publics ou privés. 


\title{
Subproblem Approach for Thin Shell Dual Finite Element Formulations
}

\author{
Vuong Q. Dang ${ }^{1}$, Patrick Dular ${ }^{1,2}$, Ruth V. Sabariego ${ }^{1}$, Laurent Krähenbühl ${ }^{3}$ and Christophe Geuzaine ${ }^{1}$ \\ ${ }^{1}$ University of Liège, Dept. of Electrical Engineering and Computer Science, ACE, B-4000 Liège, Belgium \\ ${ }^{2}$ Fonds de la Recherche Scientifique - F.R.S.-.FNRS, B-1000 Brussels, Belgium \\ ${ }^{3}$ Université de Lyon, Ampère (CNRS UMR5005), École Centrale de Lyon, F-69134 Écully Cedex, France
}

\begin{abstract}
A subproblem technique is applied on dual formulations to the solution of thin shell finite element models. Both the magnetic vector potential and magnetic field formulations are considered. The subproblem approach developed herein couples three problems: a simplified model with inductors alone, a thin region problem using approximate interface conditions, and a correction problem to improve the accuracy of the thin shell approximation, in particular near their edges and corners. Each problem is solved on its own independently defined geometry and finite element mesh.
\end{abstract}

Index Terms - Eddy current, finite element method (FEM), magnetodynamics, subproblem method (SPM), thin shell (TS).

\section{INTRODUCTION}

$\mathbf{T}$ HE solution by means of the subproblem method (SPM) provides advantages in repetitive analyses and also helps improving the overall accuracy of the solution [1], [2]. The SPM allows to benefit from previous computations instead of starting a new complete finite element (FE) solution for any variation of geometrical or physical characteristics. Furthermore, each subproblem has its own separate meshe, which increases computational efficiency.

The SPM for dual finite element $\boldsymbol{b}$ - and $\boldsymbol{h}$-formulations are herein developed within the thin shell (TS) framework $[1,3,4]$. A first problem (SP 1) involving massive or stranded inductors alone is solved on a simplified mesh without thin regions. Its solution gives surface sources (SSs) for a second problem TS (SP 2) through interface conditions (ICs), based on a 1-D approximation [3], [4]. The TS solution is then corrected in a third problem (SP 3) via SSs and VSs, that suppress the TS representation and add the actual volume, to take the field distribution of the field near edges and corners into account, which are poorly presented by the TS approximation. The method is validated on test problems with comparison with a classical FE method.

\section{Definition of the Subproblem Approach}

\section{A. Canonical magnetodynamic or static problem}

A canonical magnetodynamic or static problem $p$, to be solved at step $p$ of the subproblem approach, is defined in a domain $\Omega_{p}$, with boundary $\partial \Omega_{p}=\Gamma_{p}=\Gamma_{h, p} \cup \Gamma_{b, p}$. The eddy current conducting part of $\Omega_{p}$ is denoted $\Omega_{c, p}$ and the non-conducting region $\Omega_{c, p}^{C}$, with $\Omega_{p}=\Omega_{c, p} \cup \Omega_{c, p}^{C}$. Stranded inductors belong to $\Omega_{c, p}^{C}$, whereas massive inductors belong to $\Omega_{c, p}$. The equations, material relations and boundary conditions (BCs) of SPs $p=1,2$ and 3 are:

$$
\begin{gathered}
\operatorname{curl} \boldsymbol{h}_{p}=\boldsymbol{j}_{p}, \operatorname{div} \boldsymbol{b}_{p}=0, \operatorname{curl} \boldsymbol{e}_{p}=-\partial_{t} \boldsymbol{b}_{p}, \\
\boldsymbol{h}_{p}=\mu_{p}^{-1} \boldsymbol{b}_{p}+\boldsymbol{h}_{s, p}, \boldsymbol{b}_{p}=\mu_{p} \boldsymbol{h}_{p}+\boldsymbol{b}_{s, p}, \\
\boldsymbol{j}_{p}=\sigma_{p} \boldsymbol{e}_{p}+\boldsymbol{j}_{s, p}, \boldsymbol{e}_{p}=\sigma_{p}^{-1} \boldsymbol{j}_{p}+\boldsymbol{e}_{s, p}, \\
\boldsymbol{n} \times\left.\boldsymbol{h}_{p}\right|_{\Gamma_{h, p}}=\boldsymbol{j}_{s u, p},\left.\boldsymbol{n} \cdot \boldsymbol{b}_{p}\right|_{\Gamma_{b, p}}=\boldsymbol{b}_{s u, p}, \\
\boldsymbol{n} \times\left.\boldsymbol{e}_{p}\right|_{\Gamma_{e, p} \subset \Gamma_{b, p}}=\boldsymbol{k}_{s u, p},
\end{gathered}
$$

Manuscript received July 01, 2011. Corresponding author: Vuong Q.Dang (e-mail: quocvuong.dang@ulg.ac.be). where $\boldsymbol{h}_{p}$ is the magnetic field, $\boldsymbol{b}_{p}$ is the magnetic flux density, $\boldsymbol{e}_{p}$ is the electric field, $\boldsymbol{j}_{s, p}$ is the electric current density, $\mu_{p}$ is the magnetic permeability, $\sigma_{p}$ is the electric conductivity and $\boldsymbol{n}$ is the unit normal exterior to $\Omega_{p}$. The notation $[\cdot]_{\gamma_{p}}=\left.\right|_{\gamma_{p}^{+}}-\left.\right|_{\gamma_{p}^{-}}$ expresses the discontinuity of a quantity through an interface $\gamma_{p}$ (with sides $\gamma_{p}^{+}$and $\gamma_{p}^{-}$) in $\Omega_{p}$, defining interface conditions (ICs).

The fields $\boldsymbol{h}_{s, p}, \boldsymbol{b}_{s, p}, \boldsymbol{j}_{s, p}$ and $\boldsymbol{e}_{s, p}$ in (2a)-(2b) and (3a)(3b) respectively are VSs which can be used to account for changes of permeability or conductivity in each SP. The fields $\boldsymbol{j}_{s u, p}, \boldsymbol{b}_{s u, p}$ and $\boldsymbol{k}_{s u, p}$ in (4a-b-c) are SSs and generally equal zero for classical homogeneous BCs. Their discontinuities via ICs are also equal to zero, for common continuous field traces. If nonzero, they define possible SSs that account for particular phenomena occuring in the idealized thin region between $\gamma_{p}^{+}$ and $\gamma_{p}^{-}$[5], [7]. A typical case appears when some field traces in a previous problem are forced to be discontinuous, whereas their continuity must be recovered via a correction problem $p$; with the SSs fixed as the opposite of the trace solution of previous SP.

\section{B. From inductor alone to TS}

The constraints for SPs 1, 2 and 3 are respectively expressed via SSs and VSs. VSs are considered in Section II-C. SSs are defined via the BCs and ICs of impedance-type boundary conditions (IBC) combined with contributions from SP 1. The TS model [4] is written as a SP following the already calculated inductor source field from SP 1 . The $\boldsymbol{b}$-formulation uses a magnetic vector potential $\boldsymbol{a}$ (such that $\operatorname{curl} \boldsymbol{a}=\boldsymbol{b}$ ), written as $\boldsymbol{a}=\boldsymbol{a}_{c}+\boldsymbol{a}_{d}$ [4]. A similar analogous decomposition is done for the $\boldsymbol{h}$-formulation, with $\boldsymbol{h}=\boldsymbol{h}_{c}+\boldsymbol{h}_{d}$. Fields $\boldsymbol{a}_{c}$, $\boldsymbol{h}_{c}$ and $\boldsymbol{a}_{d}, \boldsymbol{h}_{d}$ are respectively continuous and discontinuous through the TS.

Let us analyse the SS case in both $\boldsymbol{b}$ - and $\boldsymbol{h}$-formulations as follows:

1) Constraint of SPs for b-formulation: Even if there is no thin region in SP 1, we have to forsee its future addition in order to get a relative constraint between SP 1 and SP 2 via the corresponding ICs with $\gamma_{t}=\gamma_{t}^{ \pm}=\gamma_{1}^{ \pm}=\gamma_{2}^{ \pm}$and $\boldsymbol{n}_{t}=-\boldsymbol{n}$ for the TS. One has for SPs 1 and 2 [4],

$$
\left[\boldsymbol{n} \times \boldsymbol{h}_{1}\right]_{\gamma_{1}}=\boldsymbol{n} \times\left.\boldsymbol{h}_{1}\right|_{\gamma_{1}^{+}}-\boldsymbol{n} \times\left.\boldsymbol{h}_{1}\right|_{\gamma_{1}^{-}}=0,
$$




$$
\begin{gathered}
{[\boldsymbol{n} \times \boldsymbol{h}]_{\gamma_{2}}=\left[\boldsymbol{n} \times \boldsymbol{h}_{1}\right]_{\gamma_{2}}+\left[\boldsymbol{n} \times \boldsymbol{h}_{2}\right]_{\gamma_{2}}=\sigma \beta \partial_{t}\left(2 \boldsymbol{a}_{c}+\boldsymbol{a}_{d}\right),} \\
\boldsymbol{n} \times\left.\boldsymbol{h}_{1}\right|_{\gamma_{2}}+\boldsymbol{n} \times\left.\boldsymbol{h}_{2}\right|_{\gamma_{2}+}=\frac{1}{2}\left[\sigma \beta \partial_{t}\left(2 \boldsymbol{a}_{c}+\boldsymbol{a}_{d}\right)+\frac{1}{\mu \beta} \boldsymbol{a}_{d}^{(6)}\right], \\
\beta=\gamma_{p}^{-1} \tanh \left(\frac{d_{p} \gamma_{p}}{2}\right), \gamma_{p}=\frac{1+j}{\delta_{p}}, \delta_{p}=\sqrt{2 / \omega \sigma_{p} \mu_{p}},
\end{gathered}
$$

where $d_{p}$ is the TS thickness, $\delta_{p}$ is the skin depth, $\omega=2 \pi f$, $j$ is the imaginary unit. For $\delta_{p} \gg d_{p}$, one has $\beta \approx d_{p} / 2$. In statics, (6) is equal to zero. The discontinuity $\left[\boldsymbol{n} \times \boldsymbol{h}_{1}\right]_{\gamma_{2}}$ in (6) does not need any correction because solution SP 1 presents no such discontinuity, i.e. $\left[\boldsymbol{n} \times \boldsymbol{h}_{1}\right]_{\gamma_{1}}=\left[\boldsymbol{n} \times \boldsymbol{h}_{1}\right]_{\gamma_{2}}=0$.

2) Constraint of SPs for $\boldsymbol{h}$-formulation: One gets for SPs 1 and 2 [4],

$$
\begin{gathered}
{\left[\boldsymbol{n} \times \boldsymbol{e}_{1}\right]_{\gamma_{1}}=\boldsymbol{n} \times\left.\boldsymbol{e}_{1}\right|_{\gamma_{1}^{+}}-\boldsymbol{n} \times\left.\boldsymbol{e}_{1}\right|_{\gamma_{1}^{-}}=0,} \\
{[\boldsymbol{n} \times \boldsymbol{e}]_{\gamma_{2}}=\left[\boldsymbol{n} \times \boldsymbol{e}_{1}\right]_{\gamma_{2}}+\left[\boldsymbol{n} \times \boldsymbol{e}_{2}\right]_{\gamma_{2}}=\sigma \beta \partial_{t}\left(2 \boldsymbol{h}_{c}+\boldsymbol{h}_{d}\right),} \\
\boldsymbol{n} \times\left.\boldsymbol{e}_{1}\right|_{\gamma_{2+}}+\boldsymbol{n} \times\left.\boldsymbol{e}_{2}\right|_{\gamma_{2+}}=\frac{1}{2}\left[\sigma \beta \partial_{t}\left(2 \boldsymbol{h}_{c}+\boldsymbol{h}_{d}\right)+\frac{1}{\mu \beta} \boldsymbol{h}_{d}\right] .
\end{gathered}
$$

Analogously, for $\boldsymbol{b}$-formulation in statics, (10) is equal to zero. The discontinuity $\left[\boldsymbol{n} \times \boldsymbol{e}_{1}\right]_{\gamma_{2}}$ in (10) does not need any correction because solution SP 1 verifies $\left[\boldsymbol{n} \times \boldsymbol{e}_{1}\right]_{\gamma_{2}}=0$.

\section{From TS to volume model}

The TS solution in SP 2 is next corrected by SP 3 that overcomes the TS assumptions [4]. The VSs for SP 3 are thus [1], [7],

$$
\begin{gathered}
\boldsymbol{h}_{s, 3}=\left(\mu_{3}^{-1}-\mu_{2}^{-1}\right) \boldsymbol{b}_{2}, \boldsymbol{b}_{s, 3}=\left(\mu_{3}-\mu_{2}\right) \boldsymbol{h}_{2}, \\
\boldsymbol{j}_{s, 3}=\left(\sigma_{3}-\sigma_{2}\right) \boldsymbol{e}_{2}, \boldsymbol{e}_{s, 3}=\left(\sigma_{3}^{-1}-\sigma_{2}^{-1}\right) \boldsymbol{j}_{2} .
\end{gathered}
$$

In order to correct the TS model, one has to suppress the TS representation via SSs opposed to TS ICs, and to add the actual volume shell via VSs that account for volume changes of $\mu_{p}$ and $\sigma_{p}$ from the properties of ambient region in SP 2 to these of volume shell in SP 3 (with $\mu_{2}=\mu_{0}, \mu_{3}=\mu_{\text {volume }}$, $\sigma_{2}=0$ and $\left.\sigma_{3}=\sigma_{\text {volume }}\right)$. This correction can be limited to the neighborhood of the shell, which allows to benefit from a reduction of the extension of the associated mesh [1].

\section{FINITE ELEMENT WEAK FORMULATIONS}

\section{A. Magnetic Vector Potential Formulation}

The weak $\boldsymbol{b}_{p}$-formulation is obtained from the weak form of the Ampere's law (1a), i.e. [1]-[5]. For SPs 1 and 2, they read

$$
\begin{gathered}
\left(\mu_{1}^{-1} \operatorname{curl} \boldsymbol{a}_{1}, \operatorname{curl} \boldsymbol{a}_{1}^{\prime}\right)_{\Omega_{1}}+\left\langle\boldsymbol{n} \times \boldsymbol{h}_{1}, \boldsymbol{a}_{1}^{\prime}\right\rangle_{\Gamma_{h, 1}}+\left\langle\boldsymbol{n} \times \boldsymbol{h}_{1}, \boldsymbol{a}_{1}^{\prime}\right\rangle_{\Gamma_{b, 1}} \\
+\left\langle\left[\boldsymbol{n} \times \boldsymbol{h}_{1}\right]_{\gamma_{1}}, \boldsymbol{a}_{1}^{\prime}\right\rangle_{\gamma_{1}}=\left(\boldsymbol{j}_{s, 1}, \boldsymbol{a}_{1}^{\prime}\right)_{\Omega_{1}}, \forall \boldsymbol{a}_{1}^{\prime} \in F_{1}^{1}\left(\Omega_{1}\right), \quad(14) \\
\left(\mu_{2}^{-1} \operatorname{curl} \boldsymbol{a}_{2}, \operatorname{curl} \boldsymbol{a}_{2}^{\prime}\right)_{\Omega_{2}}+\left(\sigma_{2} \partial_{t} \boldsymbol{a}_{2}, \boldsymbol{a}_{2}^{\prime}\right)_{\Omega_{2}}+\left(\sigma_{2} \operatorname{grad} v_{2}, \boldsymbol{a}_{2}^{\prime}\right)_{\Omega_{2}} \\
\left\langle\boldsymbol{n} \times \boldsymbol{h}_{2}, \boldsymbol{a}_{2}^{\prime}\right\rangle_{\Gamma_{h, 2}}+\left\langle\boldsymbol{n} \times \boldsymbol{h}_{2}, \boldsymbol{a}_{2}^{\prime}\right\rangle_{\Gamma_{b, 2}}+\left\langle\left[\boldsymbol{n} \times \boldsymbol{h}_{2}\right]_{\gamma_{2}}, \boldsymbol{a}_{2}^{\prime}\right\rangle_{\gamma_{2}} \\
=0, \forall \boldsymbol{a}_{2}^{\prime} \in F_{2}^{1}\left(\Omega_{2}\right),
\end{gathered}
$$

where $F_{p}^{1}\left(\Omega_{p}\right)$ is a gauged curl-conform function space defined on $\Omega_{p}$, gauged in $\Omega_{c, p}^{C}$, and containing the basis functions for $\boldsymbol{a}$ as well as for the test function $\boldsymbol{a}^{\prime}$ (at the discrete level, this space is defined by edge FEs; the gauge is based on the tree-cotree technique); $(\cdot, \cdot)_{\Omega}$ and $\langle\cdot, \cdot\rangle_{\Gamma}$ respectively denote a volume intergal in $\Omega$ and a surface intergal on $\Gamma$ of the product of their vector field arguments. The surface integral terms on $\Gamma_{h, p}$ account for natural BCs of type (4a), usually zero. The unknown term on the surface $\Gamma_{b, p}$ with essential BCs on $\boldsymbol{n} . \boldsymbol{b}_{p}$ is often omitted because it does not locally contribute to (14). It will be shown to be the key for the post-processing a solution, a part of which $\boldsymbol{n} \times\left.\boldsymbol{h}_{p}\right|_{\Gamma_{b, p}}$, is used as a SS in further problems [5], [7].

The term $\left\langle\left[\boldsymbol{n} \times \boldsymbol{h}_{2}\right]_{\gamma_{2}}, \boldsymbol{a}_{2}^{\prime}\right\rangle_{\gamma_{2}}$ in (15) can be rewritten as:

$$
\begin{gathered}
\left\langle\left[\boldsymbol{n} \times \boldsymbol{h}_{2}\right]_{\gamma_{2}}, \boldsymbol{a}_{2}^{\prime}\right\rangle_{\gamma_{2}}=\left\langle\left[\boldsymbol{n} \times \boldsymbol{h}_{2}\right]_{\gamma_{2}}, \boldsymbol{a}_{c}^{\prime}+\boldsymbol{a}_{d}^{\prime}\right\rangle_{\gamma_{2}}= \\
\left\langle\left[\boldsymbol{n} \times \boldsymbol{h}_{2}\right]_{\gamma_{2}}, \boldsymbol{a}_{c}^{\prime}\right\rangle_{\gamma_{2}}+\left\langle\left[\boldsymbol{n} \times \boldsymbol{h}_{2}\right]_{\gamma_{2}}, \boldsymbol{a}_{d}^{\prime}\right\rangle_{\gamma_{2}},
\end{gathered}
$$

where $\boldsymbol{a}_{d}^{\prime}$ and $\boldsymbol{a}_{c}^{\prime}$ are test functions; $\boldsymbol{a}_{d}^{\prime}$ is defined as equal to zero on the TS side $\gamma_{2}^{-}$[4]. To explicitly express the field discontinuities, (16) is also rewritten as

$$
\left\langle\left[\boldsymbol{n} \times \boldsymbol{h}_{2}\right]_{\gamma_{2}}, \boldsymbol{a}^{\prime}\right\rangle_{\gamma_{2}}=\left\langle\left[\boldsymbol{n} \times \boldsymbol{h}_{2}\right]_{\gamma_{2}}, \boldsymbol{a}_{c}^{\prime}\right\rangle_{\gamma_{2}}+\left\langle\boldsymbol{n} \times \boldsymbol{h}_{2}, \boldsymbol{a}_{d}^{\prime}\right\rangle_{\gamma_{2+}} \cdot
$$

The $\boldsymbol{h}_{p}$ trace discontinuity $\left\langle\left[\boldsymbol{n} \times \boldsymbol{h}_{2}\right]_{\gamma_{2}}, \boldsymbol{a}_{c}^{\prime}\right\rangle_{\gamma_{2}}$ in (17) is given by (6), i.e.

$\left\langle\left[\boldsymbol{n} \times \boldsymbol{h}_{2}\right]_{\gamma_{2}}, \boldsymbol{a}_{c}^{\prime}\right\rangle_{\gamma_{2}}=\left\langle[\boldsymbol{n} \times \boldsymbol{h}]_{\gamma_{2}}, \boldsymbol{a}_{c}^{\prime}\right\rangle_{\gamma_{2}}=\left\langle\sigma \beta \partial_{t}\left(2 \boldsymbol{a}_{c}+\boldsymbol{a}_{d}\right), \boldsymbol{a}_{c}^{\prime}\right\rangle_{\gamma_{2}}$.

The term $\left\langle\boldsymbol{n} \times \boldsymbol{h}_{2}, \boldsymbol{a}_{d}^{\prime}\right\rangle_{\gamma_{2}+}$ in (17) related to the positive side of the TS is given by (7), suppressing $\boldsymbol{n} \times\left.\boldsymbol{h}_{1}\right|_{\gamma_{2}^{+}}$of SP 1 and adding the actual TS BC. For that, the term $\left\langle\boldsymbol{n} \times \boldsymbol{h}_{1}, \boldsymbol{a}_{d}^{\prime}\right\rangle_{\gamma_{2}+}$ is a SS that can be naturally expressed via the weak formulation of SP 1 in (14), i.e.

$$
-\left\langle\boldsymbol{n} \times \boldsymbol{h}_{1}, \boldsymbol{a}_{d}^{\prime}\right\rangle_{\gamma_{2}^{+}}=\left(\mu_{1}^{-1} \operatorname{curl} \boldsymbol{a}_{1}, \operatorname{curl} \boldsymbol{a}_{d}^{\prime}\right)_{\Omega_{2}=\Omega_{1}} .
$$

The contribution of the volume integral in (19) is limited to a single layer of FEs on the posittive side of $\Omega_{2}^{+}=\Omega_{1}^{+}$touching $\gamma_{2}^{+}=\gamma_{1}^{+}$, because it involves only the traces $\boldsymbol{n} \times\left.\boldsymbol{a}_{d}^{\prime}\right|_{\gamma_{2}^{+}}$. At the discrete level, the source $a_{1}$, initially in mesh of SP 1 , has to be projected in mesh of SP 2 [1], [10]. The TS SP 2 solution of (15) is then corrected by SP 3 via the VSs by (10a) and (11a). Fields have also to be transferred from the mesh of TS SP 2 to the mesh of SP 3. From that, the weak form for SP 3 is

$$
\begin{gathered}
\left(\mu_{3}^{-1} \operatorname{curl} \boldsymbol{a}_{3}, \operatorname{curl} \boldsymbol{a}_{3}^{\prime}\right)_{\Omega_{3}}+\left(\sigma_{3} \partial_{t} \boldsymbol{a}_{3}, \boldsymbol{a}_{3}^{\prime}\right)_{\Omega_{c 3}}+\left\langle\boldsymbol{n} \times \boldsymbol{h}_{3}, \boldsymbol{a}_{3}^{\prime}\right\rangle_{\Gamma_{h, 3}} \\
+\left\langle\boldsymbol{n} \times \boldsymbol{h}_{3}, \boldsymbol{a}_{3}^{\prime}\right\rangle_{\Gamma_{b, 3}}+\left(\sigma_{3} \operatorname{grad} v_{3}, \boldsymbol{a}_{3}^{\prime}\right)_{\Omega_{c 3}}+\left(\boldsymbol{h}_{s, 3}, \operatorname{curl} \boldsymbol{a}_{3}^{\prime}\right)_{\Omega_{3}} \\
+\left(\boldsymbol{j}_{s, 3}, \boldsymbol{a}_{3}^{\prime}\right)_{\Omega_{c 3}}=0, \forall \boldsymbol{a}_{3}^{\prime} \in F_{3}^{1}\left(\Omega_{3}\right) .
\end{gathered}
$$

\section{B. Magnetic Field Formulation}

The weak $\boldsymbol{h}_{p}$-formulation is obtained from the weak form of the Faraday's equation (1c) [1], [7]. The field $\boldsymbol{h}_{p}$ is split into two parts, $\boldsymbol{h}_{p}=\boldsymbol{h}_{s, p}+\boldsymbol{h}_{r, p}$, where $\boldsymbol{h}_{s, p}$ is a source field defined by $\operatorname{curl} \boldsymbol{h}_{s, p}=\boldsymbol{j}_{s, p}$, and $\boldsymbol{h}_{r, p}$ is unknown. For SPs 1 and 2 , one has

$$
\begin{gathered}
\partial_{t}\left(\mu_{1} \boldsymbol{h}_{1}, \boldsymbol{h}_{1}^{\prime}\right)_{\Omega_{1}}+\partial_{t}\left(\mu_{1} \boldsymbol{h}_{s 1}, \boldsymbol{h}_{1}^{\prime}\right)_{\Omega_{1}}+\left\langle\boldsymbol{n} \times \boldsymbol{e}_{1}, \boldsymbol{h}_{1}^{\prime}\right\rangle_{\Gamma_{e, 1}}+ \\
\left\langle\left[\boldsymbol{n} \times \boldsymbol{e}_{1}\right]_{\gamma_{1}}, \boldsymbol{h}_{1}^{\prime}\right\rangle_{\gamma_{1}}=0, \forall \boldsymbol{h}_{1}^{\prime} \in F_{1}^{1}\left(\Omega_{1}\right) . \\
\partial_{t}\left(\mu_{2} \boldsymbol{h}_{2}, \boldsymbol{h}_{2}^{\prime}\right)_{\Omega_{2}}+\left(\sigma_{2}^{-1} \operatorname{curl} \boldsymbol{h}_{2}, \operatorname{curl} \boldsymbol{h}_{2}^{\prime}\right)_{\Omega_{2}}+\left\langle\boldsymbol{n} \times \boldsymbol{e}_{2}, \boldsymbol{h}_{2}^{\prime}\right\rangle_{\Gamma_{e, 2}} \\
+\left\langle\left[\boldsymbol{n} \times \boldsymbol{e}_{2}\right]_{\gamma_{2}}, \boldsymbol{h}_{c}^{\prime}\right\rangle_{\gamma_{2}}+\forall \boldsymbol{h}_{2}^{\prime} \in F_{2}^{1}\left(\Omega_{2}\right),
\end{gathered}
$$


where $F_{p}^{1}\left(\Omega_{p}\right)$ is a curl-conform function space defined on $\Omega_{p}$ and containing the basis functions for $\boldsymbol{h}$ as well as for the test function $\boldsymbol{h}^{\prime}$. The surface integral terms on $\Gamma_{e, p}$ account for natural BCs of type (4c), usually zero.

The term $\left\langle\left[\boldsymbol{n} \times \boldsymbol{e}_{2}\right]_{\gamma_{2}}, \boldsymbol{h}_{2}^{\prime}\right\rangle_{\gamma_{2}}$ in (22) expresses as:

$$
\begin{gathered}
\left\langle\left[\boldsymbol{n} \times \boldsymbol{e}_{2}\right]_{\gamma_{2}}, \boldsymbol{h}^{\prime}\right\rangle_{\gamma_{2}}\left\langle\left[\boldsymbol{n} \times \boldsymbol{e}_{2}\right]_{\gamma_{2}}, \boldsymbol{h}_{c}+\boldsymbol{h}_{d}^{\prime}\right\rangle_{\gamma_{2}} \\
=\left\langle\left[\boldsymbol{n} \times \boldsymbol{e}_{2}\right]_{\gamma_{2}}, \boldsymbol{h}_{c}^{\prime}\right\rangle_{\gamma_{2}}+\left\langle\left[\boldsymbol{n} \times \boldsymbol{e}_{2}\right]_{\gamma_{2}}, \boldsymbol{h}_{d}^{\prime}\right\rangle_{\gamma_{2}},
\end{gathered}
$$

where $\boldsymbol{h}_{d}^{\prime}$ and $\boldsymbol{h}_{c}^{\prime}$ are test functions; $\boldsymbol{h}_{d}^{\prime}$ is equal to zero on the TS side $\gamma_{2}^{-}$[4]. The field discontinuity term in (23) becomes

$$
\left\langle\left[\boldsymbol{n} \times \boldsymbol{e}_{2}\right]_{\gamma_{2}}, \boldsymbol{h}^{\prime}\right\rangle_{\gamma_{2}}=\left\langle\left[\boldsymbol{n} \times \boldsymbol{e}_{2}\right]_{\gamma_{2}}, \boldsymbol{h}_{c}^{\prime}\right\rangle_{\gamma_{2}}+\left\langle\boldsymbol{n} \times \boldsymbol{e}_{2}, \boldsymbol{h}_{d}^{\prime}\right\rangle_{\gamma_{2}+} .
$$

The $\boldsymbol{e}_{p}$ trace discontinuity $\left\langle\left[\boldsymbol{n} \times \boldsymbol{e}_{2}\right]_{\gamma_{2}}, \boldsymbol{h}_{c}^{\prime}\right\rangle_{\gamma_{2}}$ in (24) is given by (10), i.e.

$\left\langle\left[\boldsymbol{n} \times \boldsymbol{e}_{2}\right]_{\gamma_{2}}, \boldsymbol{h}_{c}^{\prime}\right\rangle_{\gamma_{2}}=\left\langle[\boldsymbol{n} \times \boldsymbol{e}]_{\gamma_{2}}, \boldsymbol{h}_{c}^{\prime}\right\rangle_{\gamma_{2}}=\left\langle\sigma \beta \partial_{t}\left(2 \boldsymbol{h}_{c}+\boldsymbol{h}_{d}\right), \boldsymbol{h}_{c}^{\prime}\right\rangle_{\gamma_{2}}$

The term $\left\langle\boldsymbol{n} \times \boldsymbol{e}_{2}, \boldsymbol{h}_{d}^{\prime}\right\rangle_{\gamma_{2}+}$ in (24) is given by (11), suppressing $\boldsymbol{n} \times\left.\boldsymbol{e}_{1}\right|_{\gamma_{2}^{+}}$of SP 1 and adding the actual TS BC. Thus, the term $\left\langle\boldsymbol{n} \times \boldsymbol{e}_{1}, \boldsymbol{h}_{d}^{\prime}\right\rangle_{\gamma_{2}}$ is a SS that can be naturally presented via the weak formulation of SP 1 in (21), i.e.

$$
-\left\langle\boldsymbol{n} \times \boldsymbol{e}_{1}, \boldsymbol{h}_{d}^{\prime}\right\rangle_{\gamma_{2}^{+}}=\left(\mu_{1} \partial_{t} \boldsymbol{h}_{s 1}, \boldsymbol{h}_{d}^{\prime}\right)_{\Omega_{1}}+\left(\mu_{1} \partial_{t} \boldsymbol{h}_{1}, \boldsymbol{h}_{d}^{\prime}\right)_{\Omega_{1}} \text {. }
$$

The contributions of the volume integrals in (26) are also limited to a single layer of FEs on the posittive side of $\Omega_{1}^{+}=\Omega_{2}^{+}$touching $\gamma_{2}^{+}=\gamma_{1}^{+}$, because it involves only the traces $\boldsymbol{n} \times\left.\boldsymbol{h}_{d}^{\prime}\right|_{\gamma_{2}^{+}}$. At the discrete level, the source $\boldsymbol{h}_{1}$, initially in mesh of SP 1, has to be projected in mesh of SP 2 [1], [10]. The inaccurate TS SP 2 solution of (22) is then corrected by SP3 via VSs by (12b) and (13b). The weak form for SP 3 is

$$
\begin{gathered}
\partial_{t}\left(\mu_{3} \boldsymbol{h}_{3}, \boldsymbol{h}_{3}^{\prime}\right)_{\Omega_{3}}+\left(\sigma_{3}^{-1} \operatorname{curl} \boldsymbol{h}_{3}, \operatorname{curl} \boldsymbol{h}_{3}^{\prime}\right)_{\Omega_{3}}+\partial_{t}\left(\boldsymbol{b}_{s, 3}, \boldsymbol{h}_{3}^{\prime}\right)_{\Omega_{3}} \\
+\left(\boldsymbol{e}_{s, 3}, \operatorname{curl} \boldsymbol{h}_{3}^{\prime}\right)_{\Omega_{3}}+\left\langle\boldsymbol{n} \times \boldsymbol{e}_{3}, \boldsymbol{h}_{3}^{\prime}\right\rangle_{\Gamma_{e 3}}=0 \\
\forall \boldsymbol{h}_{3}^{\prime} \in F_{3}^{1}\left(\Omega_{3}\right) .
\end{gathered}
$$

\section{TS Correction-VSs in the Actual Volumic Shell}

Changes of material properties from $\mu_{2}$ and $\sigma_{2}$ to $\mu_{3}$ and $\sigma_{3}$ are taken into account in (20) and (27) via the volume integrals $\left(\boldsymbol{h}_{s, 3}, \operatorname{curl} \boldsymbol{a}_{3}^{\prime}\right)_{\Omega_{3}},\left(\boldsymbol{j}_{s, 3}, \boldsymbol{a}_{3}^{\prime}\right)_{\Omega_{c 3}}$ and $\left(\boldsymbol{e}_{s, 3}, \operatorname{curl} \boldsymbol{h}_{3}^{\prime}\right)_{\Omega_{3}}$, $\partial_{t}\left(\boldsymbol{b}_{s, 3}, \boldsymbol{h}_{3}^{\prime}\right)_{\Omega_{3}}$. The VS $\boldsymbol{h}_{s, 3}$ is given by (12a), with $\boldsymbol{b}_{2}=$ curl $\boldsymbol{a}_{2}$ (at the discrete level, the source $\boldsymbol{a}_{2}$ in (20) is initially given in mesh of SP 2 and must be projected in mesh of SP 3 ). The VS $j_{s, 3}$ is given by (13a), generally reduced to $j_{s, 3}$ $=\sigma_{3} \boldsymbol{e}_{2}=\sigma_{3}\left(-\partial_{t} \boldsymbol{a}_{2}-\operatorname{grad} v_{2}\right)$. Potential $v_{2}$ can generally be fixed to zero. The VS $e_{s, 3}$ in (19b) is to be obtained from the still undetermined electric field $\boldsymbol{e}_{2}$, with $\boldsymbol{e}_{s, 3}=\left(\sigma_{2} / \sigma_{3^{-}}\right.$ 1) $e_{2}$. Indeed, the field $\boldsymbol{e}_{2}$ is unknown in $\Omega_{c, 2}^{C}$. Its determination requires to solve an electric problem defined by the Faraday and electric conservation equations, with regard to the electric constitutive relation [7].

\section{Application Example}

The first test problem is a shielded induction heater. It comprises two stranded inductors, a plate in the middle, and two screens $\left(\mu_{\text {screen }}=1, \sigma_{\text {screen }}=37.7 \mathrm{MS} / \mathrm{m}\right)(2-\mathrm{D}$, Fig. 1$)$. It is first considered via an SP 1 with the stranded inductors alone (Fig. 2, top left, $\boldsymbol{a}_{1}$ ), adding a TS FE SP 2 (Fig. 2, top right, $\boldsymbol{a}_{2}$ ) that does not include the inductor anymore. Finally, an SP 3 replaces the TS FEs with actual volume FEs (Fig. 2, bottom middle, $\boldsymbol{a}_{3}$ ). The complete solution is shown as well (Fig. 2, bottom right, $\boldsymbol{a}_{1}+\boldsymbol{a}_{2}+\boldsymbol{a}_{3}$ ). The magnetic flux density error on TS SP 2 is pointed out through the relative correction (Fig. 3, top), for different plate parameters. Significant errors can reach $85 \%$ in the end regions of the plate. Accurate local corrections are checked to be close to the complete volume FE solution by SP 3 (Fig. 3, bottom). The TS longitudinal magnetic flux and eddy current density are obtained via the relative correction as well (Fig. 4). They can reach several tens of percents in the shells, such as $60 \%$ near the screen ends (Fig. 4, top), with $\delta=0.92 \mathrm{~mm}$, or $40 \%$ (Fig. 4, bottom), with $\delta=1.59 \mathrm{~mm}$.

The second test problem is the TEAM problem 21 (model B, coil and plate, Fig. 5). The inaccuracies on the Joule power loss density of TS SP 2 are pointed by the importance of correction SP 3 (Fig. 6). The error on TS SP 2 solution along the vertical half edge ( $z$-direction) can reach $75 \%$ at the middle of the plate (Fig. 6, top), or $80 \%$ along the horizontal half inner width ( $x$-direction) (Fig. 6, bottom), with $\delta=2.975 \mathrm{~mm}$ and $d=10 \mathrm{~mm}$ in both cases. The errors diminish for a smaller thickness $(d=2 \mathrm{~mm})$, being lower than $18.85 \%$ (Fig. 6, top, bottom). Distribution of eddy current density on the TS SP 2 and in the actual volume SP 3 for $d=2 \mathrm{~mm}$ and $d=10 \mathrm{~mm}$ are depicted in Fig. 5 (from left to right).

\section{Conclusions}

The correction of inaccuracies of a TS model has been done via an SPM. Accurate eddy current, power loss density and magnetic flux distributions are successfully obtained at the edges and corners of the thin regions. All the steps of the method have been illustrated and validated with the $b$ - and $h$ formulations in $2 \mathrm{D}$ and $3 \mathrm{D}$ cases. In particular, it has been successully applied to the TEAM problem 21 .

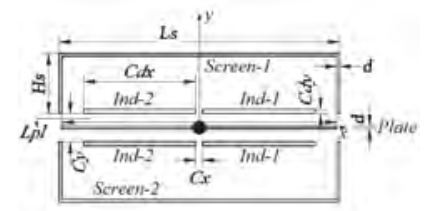

Fig. 1. Shielded induction heater $\left(d=2 \div 6 \mathrm{~mm}, L_{p l}=2 \mathrm{~m}, L_{s}=2 \mathrm{~m}+2 \mathrm{~d}\right.$ $\left., H_{s}=0.4 \mathrm{~m}, C_{d x}=0.8 \mathrm{~m}, C_{d y}=0.01 \mathrm{~m}, C_{y}=0.2 \mathrm{~m}, C_{x}=0.05 \mathrm{~m}\right)$

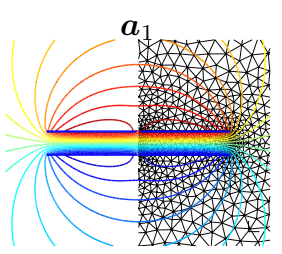

$$
\boldsymbol{a}_{\text {proj }}, \mathrm{VS}
$$

$\cdot$
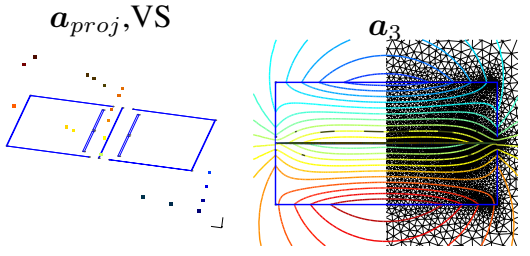

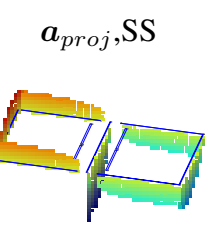

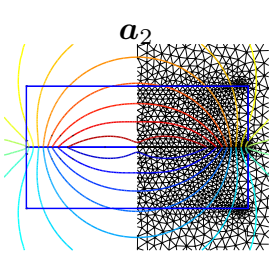

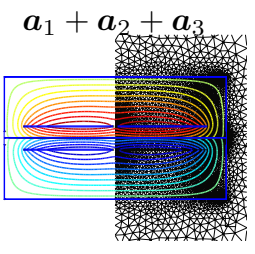

Fig. 2. Flux lines for the SP $1\left(\boldsymbol{a}_{1}\right)$, SP 2 added $\left(\boldsymbol{a}_{2}\right)$, SP 3 solution $\left(\boldsymbol{a}_{3}\right)$ and the total solution $\left(\boldsymbol{a}_{1}+\boldsymbol{a}_{2}+\boldsymbol{a}_{3}\right)$ with the different meshes used $(f=1 \mathrm{kHz}$, $\left.\mu_{\text {plate }}=100, \sigma_{\text {plate }}=1 \mathrm{MS} / \mathrm{m}\right)$. Projection of SP 1 solution $\left(\boldsymbol{a}_{\text {proj }}, \mathrm{SS}\right)$ in the SP 2, and of SP 2 solution ( $a_{\text {proj }}$, VS) in the SP 3. 

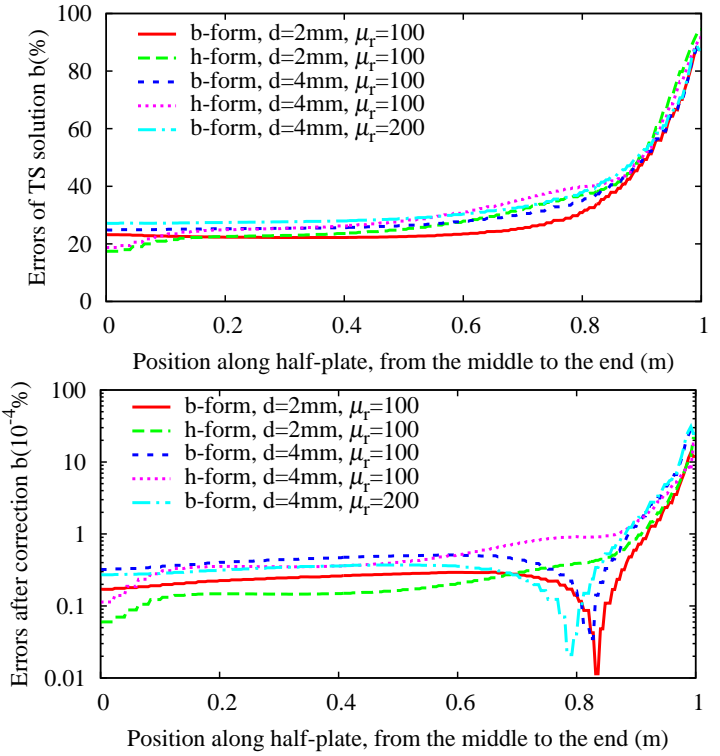

Fig. 3. The TS errors on the magnetic flux density along the plate (top) and comparison of the corrected solution (bottom) with aclassical FE volume model, with different effects of $d, \mu_{r}\left(\sigma_{\text {plate }}=1 \mathrm{MS} / \mathrm{m}, f=1 \mathrm{kHz}\right)$
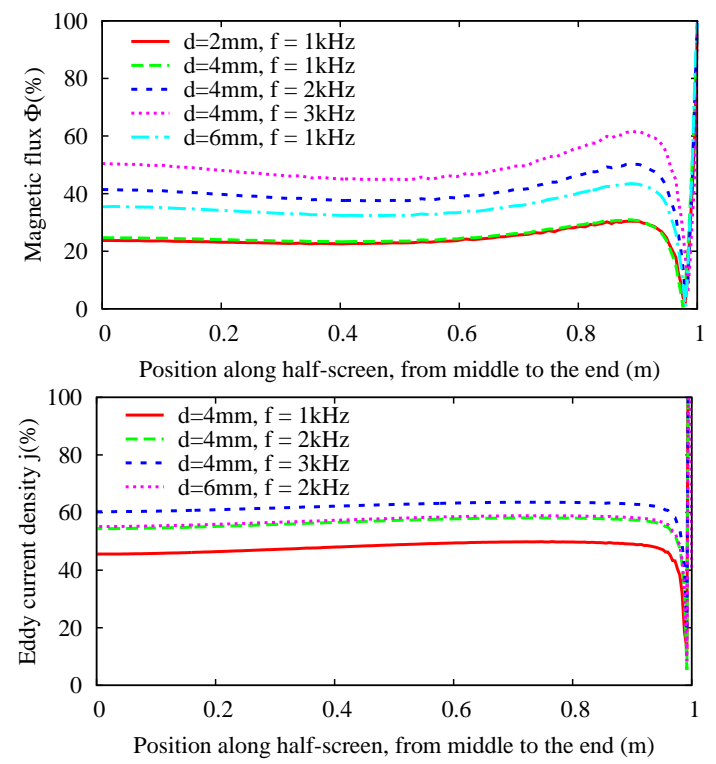

Fig. 4. Relative correction of the longitudinal magnetic flux (top) and eddy current density (bottom) along the screen for effects of $d$ and frequency $f$ $\left(\mu_{\text {plate }}=100, \sigma_{\text {plate }}=1 \mathrm{MS} / \mathrm{m}\right)$, with $b$-form.
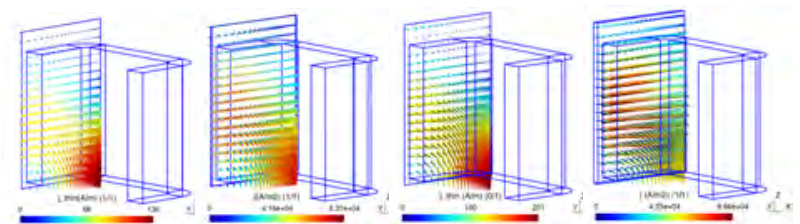

Fig. 5. TEAM problem 21 (1/4th of the geometry, magnetodynamics); eddy current density for the TS SP 2 and volume SP 3 solution (from left to right); for error reaching $18.85 \%$ with $d=2 \mathrm{~mm}$ and $77.3 \%$ with $d=10 \mathrm{~mm}(f=$ $\left.50 \mathrm{~Hz}, \mu_{\text {plate }}=100, \sigma_{\text {plate }}=6.484 \mathrm{MS} / \mathrm{m}\right)$.

\section{ACKNOWLEDGMENT}

This work was supported by the Belgian Sciency Policy (IAP P6/21).
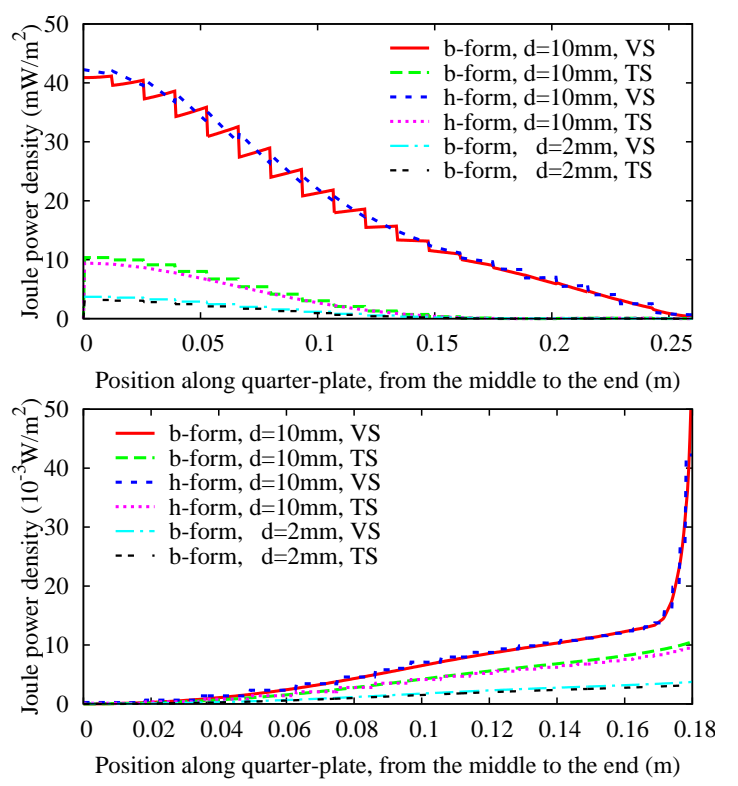

Fig. 6. Joule power loss density between TS and VS solution along vertical half edge (top) and horizontal half inner width (bottom), with effect of different thicknesses $d\left(\mu_{\text {plate }}=100, \sigma_{\text {plate }}=6.484 \mathrm{MS} / \mathrm{m}\right.$ and $\left.f=50 \mathrm{~Hz}\right)$.

\section{REFERENCES}

[1] P. Dular, Vuong Q. Dang, R. V. Sabariego, L. Krähenbühl and C. Geuzaine, "Correction of thin shell finite element magnetic models via a subproblem method," IEEE Trans. Magn., vol. 47, no. 5, pp. 158 $-1161,2011$.

[2] P. Dular, R. V Sabariego, C. Geuzaine, M. V. Ferreira da Luz, P. KuoPeng and L. Krähenbühl, "Finite Element Magnetic Models via a Coupling of Subproblems of Lower Dimensions," IEEE Trans. Magn., vol. 46, no. 8, pp. 2827-2830, 2010.

[3] L. Krähenbühl and D. Muller, "Thin layers in electrical engineering. Examples of shell models in analyzing eddy- currents by boundary and finite element methods," IEEE Trans. Magn., vol. 29, no. 2, pp. 1450$1455,1993$.

[4] C. Geuzaine, P. Dular, and W. Legros, "Dual formulations for the modeling of thin electromagnetic shells using edge elements," IEEE Trans. Magn., vol. 36, no. 4, pp. 799-802, 2000.

[5] P. Dular, R. V. Sabariego, M. V. Ferreira da Luz, P. Kuo-Peng and L. Krähenbühl, "Perturbation Finite Element Method for Magnetic Model Refinement of Air Gaps and Leakage Fluxes," IEEE Trans. Magn., vol. 45, no. 3, pp. 1400-1403, 2009.

[6] I.D. Mayergoyz and G. Bedrosian, "On calculation of 3-D eddy currents in conducting and magnetic shells," IEEE Trans. Magn., vol. 31, no. 3, pp. 1319-1324, 1995.

[7] P. Dular and R. V. Sabariego, "A perturbation method for computing field distortions due to conductive regions with h-conform magnetodynamic finite element formulations," IEEE Trans. Magn., vol. 43, no. 4, pp. 1293-1296, 2007.

[8] X. Brunotte and G. Meunier, "Line element for efficent comuptation of the magnetic field created by thin iron plates," IEEE Trans. Magn., vol. 26, no. 3, pp. 2196-2198, 1990.

[9] Z. Ren, "Degenerated Whiney prism elements-general nodal and edge shell elements for field computation in thin structures," IEEE Trans. Magn., Vol. 34, No. 05, pp.2547-2550, 1998.

[10] C. Geuzaine, B. Meys, F. Henrotte, P. Dular and W. Legros, "A Galerkin projection method for mixed finite elements," IEEE Trans. Magn., Vol. 35, No. 3, pp. 1438-1441, 1999.

[11] Tsuboi, H., Asahara, T., Kobaysashi, F. and Misaki, T. (1997), "Eddy current analysis on thin conducting plate by an integral equation method using edge elements," IEEE Trans. Magn., Vol. 33, No. 2, pp. 1346-9.

[12] C. Guerin, G. Tanneau, G. Meunier, X. Brunotte and J.B. Albertini, "Three dimensional magnetostaic finite elements for gaps and iron shells using magnetic scalar potentials," IEEE Trans. Magn., Vol. 30, No. 5, pp.2888, 1994. 